\title{
SPECTRAL INEQUALITIES FOR KUBO-ANDO OPERATOR MEANS
}

\author{
RUTE LEMOS AND GRAÇA SOARES
}

\begin{abstract}
Eigenvalue and singular value inequalities, involving Kubo-Ando operator connections or means, are established. Previous results from Lemos and Soares in 2018 are generalized or complemented, some log-majorizations are included. As a consequence, a refinement of an independent result by Ando and by Visick in 1995 on the eigenvalues of the Hadamard product is derived. Some singular value inequalities by Zou in 2017 are further extended.
\end{abstract}

\section{INTRODUCTION}

Let $M_{n}$ be the algebra of $n$-square complex matrices and $I$ be the identity matrix of order $n$. The subsets of $M_{n}$ of all Hermitian and all symmetric matrices in $M_{n}$ are denoted by $H_{n}$ and $H_{n}^{T}$, respectively. Let $A, B, X \in M_{n}$. For simplicity of notation, we consider $A_{X}=X^{*} A X$. As usual, $A \geq B$ means that $A, B \in H_{n}$ and $A-B$ is positive semidefinite, and $A>0$ means that $A$ is positive definite. The Hadamard product of $A$ and $B$ is denoted by $A \circ B$. If $A$ has real spectrum, let the eigenvalues of $A$ be arranged as follows

$$
\lambda_{1}(A) \geq \cdots \geq \lambda_{n}(A) .
$$

If $A \geq B$, then $A_{X} \geq B_{X}$ and $\lambda_{i}(A) \geq \lambda_{i}(B), i=1, \ldots, n$ [6]. We also recall that $A B$ and $B A$ have the same eigenvalues, including multiplicities [6, Theorem 1.3.20] and $\lambda_{1}\left(A^{-1}\right)=\lambda_{n}^{-1}(A)$, whenever $A$ is invertible. Let the singular values of $A$, that is the eigenvalues of $|A|$, the unique positive semidefinite square root of $A^{*} A$, be denoted and ordered as follows

$$
s_{1}(A) \geq \cdots \geq s_{n}(A) .
$$

The spectral or operator norm of $A$ is $\|A\|=s_{1}(A)$. A norm $\|\cdot\|$ on $M_{n}$ is said to be unitarily invariant if $\|A\|=\|U A V\|$ for all $A \in M_{n}$ and all $U, V \in M_{n}$ unitary. The operator norm, other Ky Fan $k$-norms and Schatten $p$-norms [2] are examples of such norms.

If $A, B$ have nonnegative eigenvalues, the log-majorization $A \prec_{\log } B$ is defined by

$$
\prod_{i=1}^{k} \lambda_{i}(A) \leq \prod_{i=1}^{k} \lambda_{i}(B), \quad k=1, \ldots, n,
$$

with equality for $k=n$. If $A, B$ have real eigenvalues, the majorization $A \prec B$ means

$$
\sum_{i=1}^{k} \lambda_{i}(A) \leq \sum_{i=1}^{k} \lambda_{i}(B), \quad k=1, \ldots, n,
$$

with equality for $k=n$; the weak majorization $A \prec_{\mathrm{w}} B$ holds if (1) is fulfilled. Log-majorization implies weak majorization and, in particular, trace inequalities. Majorization is a powerful tool to derive norm inequalities. Two classical results $[2,10]$ are:

Schur Majorization Theorem: $H \circ I \prec H$ for any $H \in H_{n}$;

Fan Dominance Theorem: $|A| \prec_{\mathrm{w}}|B| \Leftrightarrow\|A\| \leq\|B\|$ for any unitarily invariant norm $\|\cdot\|$.

Key words and phrases. eigenvalue and singular value inequalities; Hadamard product; Kubo-Ando theory; $\log$-majorization; Löwner-Heinz inequality; operator connections; operator means

2010 Mathematics Subject Classification. 15A42; 47A63; 47A64; 47A30 . 
This note is organized as follows. In Section 2, Kubo-Ando theory of operator connections and means [7] is recalled. The main theorem is proved in Section 3. Eigenvalue and singular value inequalities involving operator connections are established. Some results from $[8,14]$ are extended or complemented. For instance, in Section 4, two eigenvalue inequalities independently proved in [8] are deduced from particular cases of the main result. In Section 5, we derive some log-majorizations for weighted geometric means, using the antisymmetric tensor power technique. In Section 6, we present a refinement of the next result by Ando [1] and Visick [12]:

$$
\prod_{i=k}^{n} \lambda_{i}(A \circ B) \geq \prod_{i=k}^{n} \lambda_{i}\left(A B^{T}\right), \quad k=1, \ldots, n,
$$

for $A, B \geq 0$, inspired by a parallel result of Hiai and Lin [5]. In Section 7, singular value inequalities by Zou [14] are further generalized.

\section{Kubo-Ando THEORY OF OPERATOR CONNECTIONS AND MEANS}

The axiomatic theory of operator connections was developed by F. Kubo and T. Ando [7]. A matrix connection of order $n$ is a binary operation $\sigma$ on the cone of positive semidefinite matrices in $M_{n}$, satisfying for any $A, B, C, D, A_{k}, B_{k} \geq 0$ :

C1. (joint monotonicity) $A \leq C$ and $B \leq D \Rightarrow A \sigma B \leq C \sigma D$;

C2. (transformer inequality) $(A \sigma B)_{X} \leq A_{X} \sigma B_{X}$ for any $X \in M_{n}$;

C3. (joint continuity from above) $A_{k} \downarrow A$ and $B_{k} \downarrow B \Rightarrow A_{k} \sigma B_{k} \downarrow A \sigma B$.

If $X$ is invertible, $\mathbf{C 2}$. becomes a transformer equality and we get the positive homogeneity

$$
c(A \sigma B)=(c A) \sigma(c B), \quad c \in \mathbb{R}^{+} .
$$

An operator connection is a matrix connection of every order $n \in \mathbb{N}$. An operator mean is an operator connection $\sigma$, satisfying the normalization property $I \sigma I=I$.

If $\sigma, \tau$ are operator connections and $a, b \in \mathbb{R}^{+}$, then $a \sigma+b \tau$ is an operator connection too. A partial order is introduced on the cone of operator connections by $\sigma \leq \tau$ if and only if

$$
A \sigma B \leq A \tau B, \quad \forall A, B \geq 0 .
$$

On the core of Kubo-Ando theory is the interplay between operator connections and operator monotone functions, that is, real-valued continuous functions $f$ defined on a real interval $\Omega$, such that

$$
A \geq B \quad \Rightarrow \quad f(A) \geq f(B)
$$

for all $A, B \in H_{n}$ with spectra in $\Omega$ and all $n \in \mathbb{N}$. Löwner-Heinz inequality $[9,13]$ states that

$$
A \geq B \geq 0 \quad \Rightarrow \quad A^{\alpha} \geq B^{\alpha}, \quad \alpha \in[0,1],
$$

that is, $f(t)=t^{\alpha}, t \geq 0$, with $\alpha \in[0,1]$ is an operator monotone function. For each operator connection $\sigma$, there exists a unique operator monotone function $f: \mathbb{R}^{+} \rightarrow \mathbb{R}^{+}$, satisfying

$$
f_{\sigma}(t) I=I \sigma(t I), \quad t>0,
$$

with $f_{\sigma}(1)=1$ if $\sigma$ is an operator mean. The map $\sigma \mapsto f_{\sigma}$ is an affine order-isomorphism and $f_{\sigma}$ is called the representing function of $\sigma$. Conversely, for any operator monotone function $f: \mathbb{R}^{+} \rightarrow \mathbb{R}^{+}$, there exists a unique operator connection $\sigma$ given for $A, B>0$ by

$$
A \sigma B=A^{\frac{1}{2}} f\left(A^{-\frac{1}{2}} B A^{-\frac{1}{2}}\right) A^{\frac{1}{2}}
$$

with the right hand side defined via analytic functional calculus and extended to $A, B \geq 0$ by

$$
A \sigma B=\lim _{\epsilon \rightarrow 0^{+}}(A+\epsilon I) \sigma(B+\epsilon I) \text {. }
$$


The dual of a nonzero operator connection $\sigma$ is the operator connection $\sigma^{\perp}$ defined by

$$
A \sigma^{\perp} B=\left(B^{-1} \sigma A^{-1}\right)^{-1}
$$

for $A, B>0$ and extended by continuity to $A, B \geq 0$ as usual. Its representing function satisfies

$$
f_{\sigma^{\perp}}(t)=t / f_{\sigma}(t), \quad t>0 .
$$

Typical examples of operator connections are the sum $A+B$ and its dual, the parallel sum. If $\sigma$ is an operator mean and $f_{\sigma}^{\prime}(1)=\alpha$, then $\sigma$ is an $\alpha$-weighted operator mean. Additionally, if $\sigma$ is symmetric, that is, $A \sigma B=B \sigma A$ for all $A, B>0$, then $\sigma$ has weight $\alpha=\frac{1}{2}$.

For $\alpha \in[0,1]$ and $r, s \in[-1,1]$, we find operator monotone functions defined by

$$
f_{\alpha, r}(t)=\left(1-\alpha+\alpha t^{r}\right)^{\frac{1}{r}}, \quad t>0,
$$

where the case $r=0$ is understood as the limit of $f_{\alpha, r}(t)$ when $r \rightarrow 0$, that is, as $t^{\alpha}$; and by

$$
F_{r, s}(t)=\left(\int_{0}^{1} f_{\alpha, r}^{s}(t) d \alpha\right)^{\frac{1}{s}}, \quad t>0,
$$

also taking the limit of $F_{r, s}(t)$ when $s \rightarrow 0$ if $s=0$. In particular, $F_{0,0}(t)=\sqrt{t}$. For fixed $t>0$, $F_{r, s}(t)$ is monotone increasing on each parameter $r, s \in[-1,1]$.

For each $\alpha \in[0,1]$ and $r \in[-1,1], f_{\alpha, r}(t)$ gives rise to the $(\alpha, r)$-weighted power operator mean, here denoted by $\mathrm{m}_{\alpha, r}$. If $\alpha=0$ and $\alpha=1$, we find the left-trivial mean $A w_{l} B=A$ and the right-trivial mean $A w_{r} B=B$. The cases $r=1$ and $r=-1$ yield

$$
A \nabla_{\alpha} B=(1-\alpha) A+\alpha B \quad \text { and } \quad A !_{\alpha} B=\left((1-\alpha) A^{-1}+\alpha B^{-1}\right)^{-1},
$$

the $\alpha$-weighted versions of the arithmetic mean and harmonic mean, respectively; $r=0$ gives

$$
A \sharp_{\alpha} B=A^{\frac{1}{2}}\left(A^{-\frac{1}{2}} B A^{-\frac{1}{2}}\right)^{\alpha} A^{\frac{1}{2}},
$$

the $\alpha$-weighted geometric mean. Indexes are dropped if $\alpha=\frac{1}{2}$. In particular, the only self-dual operator connection (in the sense that $\sigma^{\perp}=\sigma$ ) is the geometric mean $A \sharp B$, which is the unique positive semidefinite solution of the Riccati equation $X A^{-1} X=B$, also characterized by

$$
A \sharp B=\max \left\{X \in H_{n}:\left[\begin{array}{cc}
A & X \\
X & B
\end{array}\right] \geq 0\right\} .
$$

We can see that $\mathrm{m}_{\alpha, r}^{\perp}=\mathrm{m}_{1-\alpha,-r}$ and $\mathrm{m}_{\alpha, r} \leq \mathrm{m}_{\alpha, s}$, whenever $-1 \leq r \leq s \leq 1$. In particular, $!_{\alpha} \leq \sharp_{\alpha} \leq \nabla_{\alpha}$ is the arithmetic-geometric-harmonic operator mean inequality.

For $r, s \in[-1,1]$, the extension $\sigma_{r, s}$ of the power difference mean [11] is the operator mean with representing function $F_{r, s}(t)$, which is symmetric and $\sigma_{r, s}^{\perp}=\sigma_{-r,-s}$. This family interpolates well-known operator means. For instance, the logarithmic mean $\ell$ and the identric mean $\iota$ have representing functions

$$
F_{0,1}(t)=\int_{0}^{1} t^{\alpha} d \alpha=\frac{t-1}{\log t} \quad \text { and } \quad F_{1,0}(t)=\exp \left(\frac{t \log t}{t-1}-1\right), \quad t>0,
$$

respectively, and $\sharp \leq \ell \leq \iota \leq \nabla$ holds.

\section{MAIN RESULT}

Our main theorem presents spectral inequalities, dealing with matrices $A, B \geq 0$, an Hermitian or symmetric matrix $C \in M_{n}$ and nonzero operator connections $\sigma, \tau, \rho$ with representing functions, satisfying either

$$
f_{\sigma}^{2}(t) \leq f_{\tau}(t) f_{\rho}(t), \quad t>0,
$$

or the reverse inequality. It is presented in Theorem 3.1 in a condensed form that can be 
splitted into cases, depending on $C$. Under the hypothesis (4), these cases are as follows.

i. If $C$ is Hermitian, then

$$
\begin{aligned}
& \left(\mathbf{a}_{1}\right) s_{1}\left(\left(A \tau^{\perp} B\right)^{\frac{1}{2}} C(A \sigma B) C\left(A \rho^{\perp} B\right)^{\frac{1}{2}}\right) \leq \lambda_{1}(A C B C) ; \\
& \left(\mathbf{b}_{1}\right) s_{n}\left((A \tau B)^{\frac{1}{2}} C\left(A \sigma^{\perp} B\right) C(A \rho B)^{\frac{1}{2}}\right) \geq \lambda_{n}(A C B C) .
\end{aligned}
$$

ii. If $C$ is symmetric, then

$$
\begin{aligned}
& \left(\mathbf{a}_{2}\right) s_{1}\left(\left(A \tau^{\perp} B\right)^{\frac{1}{2}} \bar{C}(A \sigma B)^{T} C\left(A \rho^{\perp} B\right)^{\frac{1}{2}}\right) \leq \lambda_{1}\left(A \bar{C} B^{T} C\right) ; \\
& \left(\mathbf{b}_{2}\right) s_{n}\left((A \tau B)^{\frac{1}{2}} \bar{C}\left(A \sigma^{\perp} B\right)^{T} C(A \rho B)^{\frac{1}{2}}\right) \geq \lambda_{n}\left(A \bar{C} B^{T} C\right) .
\end{aligned}
$$

In the sequel, the condensed notation $X^{\sim}$ stands for $X$ or $X^{T}, C \in H_{n}^{\sim}$ means that $C \in H_{n}$ or $C \in H_{n}^{T}$, so that the symbol $\sim$ is either omitted or acts as ${ }^{T}$ along each result; if the symbol appears in an inequality not containing $C$, then it holds for both choices of $\sim$. This notation allows us, for instance, to present both $\left(\mathrm{a}_{1}\right)$ and $\left(\mathrm{a}_{2}\right)$ stated before as item (a) in Theorem 3.1.

Theorem 3.1. Let $A, B \geq 0$ and $C \in H_{n}^{\sim}$. Consider nonzero operator connections $\sigma, \tau, \rho$. If $f_{\sigma}^{2}(t) \leq f_{\tau}(t) f_{\rho}(t), t>0$, then

(a) $s_{1}\left(\left(A \tau^{\perp} B\right)^{\frac{1}{2}} C^{*}(A \sigma B)^{\sim} C\left(A \rho^{\perp} B\right)^{\frac{1}{2}}\right) \leq \lambda_{1}\left(A C^{*} B^{\sim} C\right)$;

(b) $s_{n}\left((A \tau B)^{\frac{1}{2}} C^{*}\left(A \sigma^{\perp} B\right)^{\sim} C(A \rho B)^{\frac{1}{2}}\right) \geq \lambda_{n}\left(A C^{*} B^{\sim} C\right)$.

If $f_{\sigma}^{2}(t) \geq f_{\tau}(t) f_{\rho}(t), t>0$, then (a) and (b) hold with each connection replaced by its dual.

Proof. Firstly, if $f_{\sigma}^{2}(t) \leq f_{\tau}(t) f_{\rho}(t), t>0$, we will prove (a) and (b) when $\sim$ is replaced by ${ }^{T}$ and $C$ is a real diagonal matrix $D$. In this case, (a) is given by

$$
s_{1}\left(\left(A \tau^{\perp} B\right)^{\frac{1}{2}} D(A \sigma B)^{T} D\left(A \rho^{\perp} B\right)^{\frac{1}{2}}\right) \leq \lambda_{1}\left(A D B^{T} D\right) .
$$

We only need to show that

$$
\lambda_{1}\left(A D B^{T} D\right) \leq 1 \Rightarrow s_{1}\left(\left(A \tau^{\perp} B\right)^{\frac{1}{2}} D(A \sigma B)^{T} D\left(A \rho^{\perp} B\right)^{\frac{1}{2}}\right) \leq 1,
$$

because both hand sides of (5) have the same order of homogeneity for $A, B, D$, and so each matrix can be multiplied by a positive scalar. If $\lambda_{1}\left(A D B^{T} D\right) \leq 1$, equivalently, $\lambda_{1}\left(D A^{T} D B\right) \leq 1$, then $D A^{T} D \leq B^{-1}$ and $D B^{T} D \leq A^{-1}$, whenever $A, B$ are invertible. By the transformer inequality and joint monotonicity of $\rho^{\perp}$, also recalling the expression of the dual, we find

$$
\begin{aligned}
D\left(A \rho^{\perp} B\right)^{T} D=D\left(A^{T} \rho^{\perp} B^{T}\right) D & \leq\left(D A^{T} D\right) \rho^{\perp}\left(D B^{T} D\right) \\
& \leq B^{-1} \rho^{\perp} A^{-1}=(A \rho B)^{-1} .
\end{aligned}
$$

Analogously, for $\tau^{\perp}$ we get $D\left(A \tau^{\perp} B\right)^{T} D \leq(A \tau B)^{-1}$. Moreover, the representing functions of $\sigma, \tau, \rho$ satisfy $f_{\sigma}^{2}(t) \leq f_{\tau}(t) f_{\rho}(t), t>0$, if and only if

$$
(A \sigma B)(A \rho B)^{-1}(A \sigma B)=A^{\frac{1}{2}} f_{\sigma}(H)\left(f_{\rho}(H)\right)^{-1} f_{\sigma}(H) A^{\frac{1}{2}} \leq A^{\frac{1}{2}} f_{\tau}(H) A^{\frac{1}{2}}=A \tau B,
$$

where $H=A^{-\frac{1}{2}} B A^{-\frac{1}{2}}$. Therefore

$$
\begin{aligned}
s_{1}^{2}\left(\left(A \tau^{\perp} B\right)^{\frac{1}{2}} D(A \sigma B)^{T} D\left(A \rho^{\perp} B\right)^{\frac{1}{2}}\right) & =\lambda_{1}\left((A \sigma B) D\left(A \rho^{\perp} B\right)^{T} D(A \sigma B) D\left(A \tau^{\perp} B\right)^{T} D\right) \\
& \leq \lambda_{1}\left((A \sigma B)(A \rho B)^{-1}(A \sigma B)(A \tau B)^{-1}\right) \leq 1
\end{aligned}
$$

and (6) holds. If $A, B \geq 0$ are not invertible, we can replace these matrices in (5) by $A+\epsilon I, B+\epsilon I>0, \epsilon>0$, and then we use a continuity argument, letting $\epsilon \downarrow 0$.

Next, we prove (b) for $A, B>0$ and $D$ invertible, otherwise $\lambda_{n}(A D B D)=0$ and the result is trivial. For that, replace $A, B, D$ in (5) by $B^{-1}, A^{-1}, D^{-1}$, respectively, and take inverses of both hand sides of this inequality, so that

$$
s_{1}^{-1}\left(\left(B^{-1} \rho^{\perp} A^{-1}\right)^{\frac{1}{2}} D^{-1}\left(B^{-1} \sigma A^{T}\right)^{-1} D^{-1}\left(B^{-1} \tau^{\perp} A^{-1}\right)^{\frac{1}{2}}\right) \geq \lambda_{1}^{-1}\left(B^{-1} D^{-1}\left(A^{T}\right)^{-1} D^{-1}\right) .
$$


Denoting the inverses of $X^{T}, X^{1 / 2}$ by $X^{-T}, X^{-\frac{1}{2}}$, the previous inequality is equivalent to

$$
s_{n}\left(\left(B^{-1} \tau^{\perp} A^{-1}\right)^{-\frac{1}{2}} D\left(B^{-1} \sigma A^{-1}\right)^{-T} D\left(B^{-1} \rho^{\perp} A^{-1}\right)^{-\frac{1}{2}}\right) \geq \lambda_{n}\left(D A^{T} D B\right)=\lambda_{n}\left(A D B^{T} D\right)
$$

and, due to the expression of the dual operator connection, we find (b) for $C=D$.

Furthermore, all the arguments above remain valid if the symbols of transpose are deleted.

Now, if $C \in H_{n}^{\sim}$, we split the proof into two cases: i. $C \in H_{n}$; ii. $C \in H_{n}^{T}$.

i. If $C$ is Hermitian, then there exists $U$ unitary, such that $U^{*} C U=D$ is real diagonal. Since $U$ is invertible, the transformer equality holds for $\sigma, \tau^{\perp}, \rho^{\perp}$ when $X=U$. After replacing $A, B$ by $A_{U}, B_{U} \geq 0$, respectively, in (5) with ${ }^{T}$ deleted, its left hand side may be written as

$$
\lambda_{1}^{\frac{1}{2}}\left((A \sigma B)_{U} D\left(A \tau^{\perp} B\right)_{U} D(A \sigma B)_{U} D\left(A \rho^{\perp} B\right)_{U} D\right)=s_{1}\left(\left(A \tau^{\perp} B\right)^{\frac{1}{2}} C(A \sigma B) C\left(A \rho^{\perp} B\right)^{\frac{1}{2}}\right) .
$$

It is also clear that $\lambda_{1}\left(A_{U} D B_{U} D\right)=\lambda_{1}(A C B C)$. Then (a) and analogously (b) follow.

ii. If $C$ is symmetric, by Takagi's factorization [6, Corollary 4.4.4], there exist $V$ unitary and $D$ diagonal, with the singular values of $C$ in the main diagonal, such that $C=V D V^{T}$. Since $\bar{V}$ is invertible, $A_{\bar{V}} \sigma B_{\bar{V}}=(A \sigma B)_{\bar{V}}$. Its transpose is $(A \sigma B)_{V}^{T}$. Replacing $A, B$ by $A_{\bar{V}}, B_{\bar{V}} \geq 0$ in (5), respectively, by the transformer equality, we get

$$
s_{1}\left(\left(\left(A \tau^{\perp} B\right)_{\bar{V}}\right)^{\frac{1}{2}} D(A \sigma B)_{V}^{T} D\left(\left(A \rho^{\perp} B\right)_{\bar{V}}\right)^{\frac{1}{2}}\right) \leq \lambda_{1}\left(A_{\bar{V}} D B_{V}^{T} D\right),
$$

where $B_{V}^{T}=\left(B_{\bar{V}}\right)^{T}$. The left hand side of the previous inequality is equal to the square root of

$$
\lambda_{1}\left(\left(A \tau^{\perp} B\right) \bar{V} D V^{*}(A \sigma B)^{T} V D V^{T}\left(A \rho^{\perp} B\right) \bar{V} D V^{*}(A \sigma B)^{T} V D V^{T}\right)
$$

and the right hand side is equal to $\lambda_{1}\left(A \bar{C} B^{T} C\right)$. Thus, (a) and similarly (b) are obtained.

Finally, if the inequality satisfied by the representing functions of $\sigma, \tau, \rho$ is reversed, then

$$
\left(t / f_{\sigma}(t)\right)^{2} \leq\left(t / f_{\tau}(t)\right)\left(t / f_{\rho}(t)\right) \quad \Leftrightarrow \quad f_{\sigma^{\perp}}^{2}(t) \leq f_{\tau^{\perp}}(t) f_{\rho^{\perp}}(t),
$$

which provides an inequality for the representing functions of the corresponding dual operator connections. Hence, the result readily follows, replacing $\sigma, \tau, \rho$ in (a) and (b) by their duals.

Under the hypothesis of Theorem 3.1, we observe that

$$
\lambda_{i}\left(A C^{*} B^{\sim} C\right)=\lambda_{i}\left(A C^{*} B^{\sim} C\right)^{\sim}=\lambda_{i}\left(B C^{*} A^{\sim} C\right), \quad i=1, \ldots, n,
$$

because $C$ is Hermitian when $\sim$ is omitted, and $C$ is symmetric when $\sim$ acts as the transpose.

Remark 3.2. If $A, B>0$, then (4) is equivalent to (7) and by [3, Theorem 1.3.3] to

$$
\left[\begin{array}{ll}
A \tau B & A \sigma B \\
A \sigma B & A \rho B
\end{array}\right] \geq 0
$$

This implies, having in mind (3), that $A \sigma B \leq(A \tau B) \sharp(A \rho B)$. The previous right hand side is equal to the composition operator mean $A \tau(\sharp) \rho B$ (see [1]). The square of its representing function

$$
f_{\tau(\sharp) \rho}(t)=f_{\tau}(t)\left(f_{\rho}(t) / f_{\tau}(t)\right)^{\frac{1}{2}}, \quad t>0,
$$

reduces to $f_{\tau}(t) f_{\rho}(t)$. In other words, the condition (4) is equivalent to $\sigma \leq \tau(\sharp) \rho$.

The reverse condition $f_{\sigma}^{2}(t) \geq f_{\tau}(t) f_{\rho}(t), t>0$, is equivalent to (8), to the positive semidefiniteness of the block matrix in (10) with $\sigma, \tau, \rho$ replaced by their duals and to $\sigma \geq \tau(\sharp) \rho$.

For $A, B \geq 0$ and $X \in M_{n}$, we have $A^{1 / 2} B_{X} A^{1 / 2} \geq 0$, so that

$$
\lambda_{1}\left(A B_{X}\right) \leq\left\|A B_{X}\right\| \quad \text { and } \quad \lambda_{n}\left(A B_{X}\right) \geq s_{n}\left(A B_{X}\right) .
$$

Thus, under the hypothesis of Theorem 3.1, if $f_{\sigma}^{2}(t) \leq f_{\tau}(t) f_{\rho}(t), t>0$, it follows that

$$
\begin{gathered}
\left\|\left(A \tau^{\perp} B\right)^{\frac{1}{2}} C^{*}(A \sigma B)^{\sim} C\left(A \rho^{\perp} B\right)^{\frac{1}{2}}\right\| \leq\left\|A C^{*} B^{\sim} C\right\|, \\
s_{n}\left((A \tau B)^{\frac{1}{2}} C^{*}\left(A \sigma^{\perp} B\right)^{\sim} C(A \rho B)^{\frac{1}{2}}\right) \geq s_{n}\left(A C^{*} B^{\sim} C\right)
\end{gathered}
$$


and analogous inequalities hold for the dual connections, whenever $f_{\sigma}^{2}(t) \geq f_{\tau}(t) f_{\rho}(t), t>0$.

\section{SOME CONSEQUENCES}

Let $A, B \geq 0, X \in M_{n}$ and $\sigma, \tau$ be nonzero operator connections, satisfying $\sigma \leq \tau$. Then $\tau^{\perp} \leq \sigma^{\perp}$ and we easily find

$$
\lambda_{j}\left(\left(A \tau^{\perp} B\right)(A \sigma B)_{X}\right) \leq \lambda_{j}\left(\left(A \sigma^{\perp} B\right)(A \sigma B)_{X}\right) \leq \lambda_{j}\left(\left(A \sigma^{\perp} B\right)(A \tau B)_{X}\right)
$$

for $j=1, \ldots, n$, as well as

$$
\operatorname{det}\left(\left(A \tau^{\perp} B\right)(A \sigma B)_{X}\right) \leq \operatorname{det}\left(A B_{X}\right) \leq \operatorname{det}\left(\left(A \sigma^{\perp} B\right)(A \tau B)_{X}\right)
$$

with equality when $\tau=\sigma$. Nevertheless, under the previous conditions,

$$
\lambda_{1}\left(\left(A \tau^{\perp} B\right)(A \sigma B)_{X}\right) \leq \lambda_{1}\left(A B_{X}\right)
$$

does not always hold, as the next counterexample shows, but it becomes true when $X$ is replaced by any $C \in H_{n}$, as shown in Corollary 4.2 .

Example 4.1. Consider

$$
A=\left[\begin{array}{cc}
1 & -1 \\
-1 & 2
\end{array}\right], \quad B=\left[\begin{array}{cc}
2 & -1 \\
-1 & 1
\end{array}\right], \quad X=\left[\begin{array}{cc}
2 & 0 \\
2 & -2
\end{array}\right]
$$

In this case $A, B \geq 0$ and

$$
\lambda_{1}\left((A \sharp B)(A \sharp B)_{X}\right) \approx 14.0622 \geq \lambda_{1}\left(A B_{X}\right) \approx 10.4721 .
$$

Some consequences of Theorem 3.1 are now provided. Relevant particular cases allow us to recover previous results from [8].

Corollary 4.2. Let $A, B \geq 0, C \in H_{n}^{\sim}$ and $\sigma, \tau$ be nonzero operator connections. If $\sigma \leq \tau$, then

$$
\begin{aligned}
& \lambda_{1}\left(\left(A \tau^{\perp} B\right) C^{*}(A \sigma B)^{\sim} C\right) \leq \lambda_{1}\left(A C^{*} B^{\sim} C\right) \\
& \lambda_{n}\left((A \tau B) C^{*}\left(A \sigma^{\perp} B\right)^{\sim} C\right) \geq \lambda_{n}\left(A C^{*} B^{\sim} C\right) .
\end{aligned}
$$

Proof. If $\sigma \leq \tau$, then $f_{\sigma}^{2}(t) \leq f_{\tau}^{2}(t), t>0$. So we may apply Theorem 3.1 with $\rho=\tau$. Since

$$
s_{1}\left(\left(A \tau^{\perp} B\right)^{\frac{1}{2}} C^{*}(A \sigma B)^{\sim} C\left(A \tau^{\perp} B\right)^{\frac{1}{2}}\right)=\lambda_{1}\left(\left(A \tau^{\perp} B\right) C^{*}(A \sigma B)^{\sim} C\right),
$$

then (12) readily follows. The other inequality is similarly obtained.

As the next counterexample shows, Corollary 4.2 is not true, keeping in the inequalities $\sim$ as the transpose, when $C \in H_{n}^{T}$ is replaced by $X \in H_{n}$.

Example 4.3. Let

$$
A=\left[\begin{array}{cc}
3 & 1-3 i \\
1+3 i & 4
\end{array}\right], \quad B=\left[\begin{array}{cc}
3 & -1+3 i \\
-1-3 i & 4
\end{array}\right], \quad X=\left[\begin{array}{cc}
-1 & -1-i \\
-1+i & -1
\end{array}\right]
$$

We have $A, B \geq 0, X \in H_{2}$ and

$$
\lambda_{1}\left((A \sharp B)(A \sharp B)_{X}^{T}\right) \approx 11.8285 \geq \lambda_{1}\left(A B_{X}^{T}\right)=4 .
$$

Remark 4.4. In particular, if $C \geq 0$ and $\tau=\sigma$ in Corollary 4.2, then [8, Theorem 2.1] is obtained from (12) when $\sim$ is not present. Reciprocally, $\sigma \leq \tau$ implies $\tau^{\perp} \leq \sigma^{\perp}$ and

$$
\lambda_{1}\left(\left(A \tau^{\perp} B\right) C(A \sigma B) C\right) \leq \lambda_{1}\left((A \sigma B) C\left(A \sigma^{\perp} B\right) C\right) \leq \lambda_{1}(A C B C)
$$

for $A, B, C \geq 0$, that is, in this special case, (12) also follows from [8, Theorem 2.1]. 
Corollary 4.5. Let $A, B \geq 0, C \in H_{n}^{\sim}$ and $\sigma$ be a nonzero operator connection. If $\sigma \leq \sigma^{\perp}$, then

$$
\begin{gathered}
\lambda_{1}\left((A \sigma B) C^{*}(A \sigma B)^{\sim} C\right) \leq s_{1}\left(A^{\frac{1}{2}} C^{*}(A \sigma B)^{\sim} C B^{\frac{1}{2}}\right) \leq \lambda_{1}\left(A C^{*} B^{\sim} C\right) ; \\
\lambda_{n}\left(\left(A \sigma^{\perp} B\right) C^{*}\left(A \sigma^{\perp} B\right)^{\sim} C\right) \geq s_{n}\left(A^{\frac{1}{2}} C^{*}\left(A \sigma^{\perp} B\right)^{\sim} C B^{\frac{1}{2}}\right) \geq \lambda_{n}\left(A C^{*} B^{\sim} C\right) .
\end{gathered}
$$

Proof. The first inequality in (13) and (14) follow from Corollary 4.2 with $\sim$ deleted, $\tau=\sigma^{\perp}$ and $C$ replaced by $C^{*}(A \sigma B)^{\sim} C \geq 0$, then taking square roots of the obtained eigenvalues. In the previous case, the right hand side becomes

$$
\lambda_{i}^{\frac{1}{2}}\left(A C^{*}(A \sigma B)^{\sim} C B C^{*}(A \sigma B)^{\sim} C\right)=s_{i}\left(A^{\frac{1}{2}} C^{*}(A \sigma B)^{\sim} C B^{\frac{1}{2}}\right), \quad i=1, n .
$$

The remaining inequalities follow from Theorem 3.1 when $\tau, \rho$ are the trivial operator means $w_{l}, w_{r}$, because $f_{w_{l}}(t) f_{w_{r}}(t)=t, t>0$, and $\sigma \leq \sigma^{\perp}$ is equivalent to $f_{\sigma}^{2}(t) \leq t, t>0$.

Taking squares of both hand sides of the second inequality in (13) with $C=I$ yields

$$
\lambda_{1}\left(A(A \sigma B)^{\sim} B(A \sigma B)^{\sim}\right) \leq \lambda_{1}^{2}\left(A B^{\sim}\right),
$$

whenever $A, B \geq 0$ and $\sigma \leq \sharp$. Having also in mind (11), the previous inequality for $\sigma=\sharp$, implies [8, (10) of Theorem 3.3] as stated in the next corollary.

Corollary 4.6. If $A, B \geq 0$, then

$$
\lambda_{1}(A(A \sharp B) B(A \sharp B)) \leq \lambda_{1}\left(A^{2} B^{2}\right) .
$$

We have just seen that Theorem 3.1 simultaneously allows us to obtain two results independently proved in [8], namely [8, Theorem 2.1], as stated in Remark 4.4, and the previous corollary.

\section{LOG-MAJORIZATIONS}

Let $k \in\{1, \ldots, n\}$ and $n_{k}=\left(\begin{array}{l}n \\ k\end{array}\right)$. The $k$ th compound or $k$ th antisymmetric tensor power of $A \in M_{n}$ is the matrix $A^{\wedge k} \in M_{n_{k}}$ with entries given by the minors $\operatorname{det} A(\mathbf{i}, \mathbf{j})$, where the index sets $\mathbf{i}, \mathbf{j} \subset\{1, \ldots, n\}$ have cardinality $k$ and are lexicographically ordered. As usual, $A(\mathbf{i}, \mathbf{j})$ denotes the submatrix of $A$ that lies in rows and columns indexed, respectively, by $\mathbf{i}$ and $\mathbf{j}$. Some useful properties [2] are listed below.

P1. $(A B)^{\wedge k}=A^{\wedge k} B^{\wedge k}, A, B \in M_{n}$ (Binet-Cauchy formula);

P2. $\left(A^{\wedge k}\right)^{T}=\left(A^{T}\right)^{\wedge k}, \overline{A^{\wedge k}}=\bar{A}^{\wedge k},\left(A^{\wedge k}\right)^{r}=\left(A^{r}\right)^{\wedge k}, r>0$ (and $r=-1$ if $A$ is invertible);

P3. The $n_{k}$ eigenvalues of $A^{\wedge k}$ are $\lambda_{i_{1}}(A) \lambda_{i_{2}}(A) \cdots \lambda_{i_{k}}(A), 1 \leq i_{1}<\cdots<i_{k} \leq n$;

P4. If $A, B \in M_{n}$ have nonnegative spectra, then

$$
A \prec_{\log } B \quad \Leftrightarrow \quad \lambda_{1}\left(A^{\wedge k}\right) \leq \lambda_{1}\left(B^{\wedge k}\right), \quad k=1, \ldots, n, \quad \text { and } \operatorname{det} A=\operatorname{det} B .
$$

Briefly, any expression involving products, fractional matrix powers and transposes "commutes" with the $k$ th compound. A powerful tool to derive matrix log-majorizations is thus provided. For instance, replacing $A, B, X$ by $A^{\wedge k}, B^{\wedge k}, X^{\wedge k}$ in the first inequality in (11) gives

$$
A B_{X} \prec_{\log }\left|A B_{X}\right|, \quad A, B \geq 0, \quad X \in M_{n} .
$$

Some log-majorizations are now derived from the results of the previous sections, applying the antisymmetric tensor power technique, also called Weyl's trick.

Corollary 5.1. If $A, B \geq 0, C \in H_{n}^{\sim}$ and $0 \leq \alpha \leq \beta \leq 1$, then

$$
\left|\left(A \sharp_{1-\alpha} B\right)^{\frac{1}{2}} C^{*}\left(A \sharp_{\frac{\beta}{2}} B\right)^{\sim} C\left(A \sharp_{1+\alpha-\beta} B\right)^{\frac{1}{2}}\right| \prec_{\log } A C^{*} B^{\sim} C .
$$


Proof. We remark that $\beta-\alpha \in[0,1]$. Consider $\sigma=\sharp_{\frac{\beta}{2}}, \tau=\sharp_{\alpha}, \rho=\sharp_{\beta-\alpha}$, with representing functions, satisfying $f_{\sigma}^{2}(t)=f_{\tau}(t) f_{\rho}(t), t>0$. By Theorem 3.1, we have

$$
s_{1}\left(\left(A \sharp_{1-\alpha} B\right)^{\frac{1}{2}} C^{*}\left(A \sharp_{\frac{\beta}{2}} B\right)^{\sim} C\left(A \sharp_{1+\alpha-\beta} B\right)^{\frac{1}{2}}\right) \leq \lambda_{1}\left(A C^{*} B^{\sim} C\right) .
$$

Then replace $A, B, C$ by their $k$ th compounds and apply properties P1-P4. For example, use

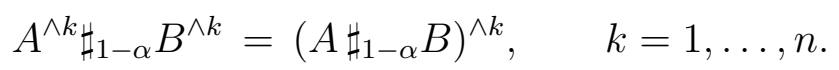

Clearly, $\operatorname{det}\left(A \sharp_{1-\alpha} B\right)=\operatorname{det}\left(A^{\alpha} B^{1-\alpha}\right)$ and the equality of the determinants of the matrices in both hand sides of (16) occurs. Thus, the log-majorization is obtained.

Under the hypothesis of Corollary 5.1, after interchanging $A$ and $B$ in (16), recalling (9) and observing that $B \sharp_{1+\alpha-\beta} A=A \sharp_{\beta-\alpha} B$, we also have

$$
\left|\left(A \sharp_{\alpha} B\right)^{\frac{1}{2}} C^{*}\left(A \sharp_{1-\frac{\beta}{2}} B\right)^{\sim} C\left(A \sharp_{\beta-\alpha} B\right)^{\frac{1}{2}}\right| \prec_{\log } A C^{*} B^{\sim} C .
$$

Corollary 5.2. If $A, B \geq 0, C \in H_{n}^{\sim}$ and $\sigma$ is a nonzero operator connection, then

$$
(A \sharp B) C^{*}(A \sharp B)^{\sim} C \prec_{\log }\left|\left(A \sigma^{\perp} B\right)^{\frac{1}{2}} C^{*}(A \sharp B)^{\sim} C(A \sigma B)^{\frac{1}{2}}\right| \prec_{\log }\left(A \sigma^{\perp} B\right) C^{*}(A \sigma B)^{\sim} C .
$$

Proof. Apply Weyl's trick to (13) when $\sigma=\sharp$, replacing $A, B, C$ by their $k$ th compounds and using properties P1-P4. Then we obtain

$$
(A \sharp B) C^{*}(A \sharp B)^{\sim} C \prec_{\log }\left|A^{\frac{1}{2}} C^{*}(A \sharp B)^{\sim} C B^{\frac{1}{2}}\right| \prec_{\log } A C^{*} B^{\sim} C,
$$

having also in mind the equality of the determinants for the three matrices in (17). Finally, replace $A$ by $A \sigma^{\perp} B, B$ by $A \sigma B$ in (17) and use the following identity $\left(A \sigma^{\perp} B\right) \sharp(A \sigma B)=$ $A \sharp B$.

Remark 5.3. The last log-majorization in (17) with $\sim$ omitted and (15) yield

$$
\left|A^{\frac{1}{2}}(A \sharp B)_{C} B^{\frac{1}{2}}\right| \prec_{\log }\left|A B_{C}\right|, \quad A, B \geq 0, \quad C \in H_{n} .
$$

If $C=I$, this is [8, Corollary 3.4], which also includes [14, Theorem 2.10] to be extended in another direction in the last section.

Let $A, B \geq 0, C \in H_{n}^{\sim}$ and $\alpha \in[0,1]$. If $\sigma=\sharp_{\alpha}$ in Corollary 5.2 and $\beta=1$ in (16), then

$$
\left|\left(A \sharp_{1-\alpha} B\right)^{\frac{1}{2}} C^{*}(A \sharp B)^{\sim} C\left(A \sharp_{\alpha} B\right)^{\frac{1}{2}}\right|
$$

is log-majorized by $\left(A \sharp_{1-\alpha} B\right) C^{*}\left(A \sharp_{\alpha} B\right)^{\sim} C$ and $A C^{*} B^{\sim} C$, respectively, being these two last matrices related as follows.

Corollary 5.4. Let $A, B \geq 0, C \in H_{n}^{\sim}$ and $\alpha \in[0,1]$. Then

$$
\left(A \sharp_{1-\alpha} B\right) C^{*}\left(A \sharp_{\alpha} B\right)^{\sim} C \prec_{\log } A C^{*} B^{\sim} C .
$$

Proof. Apply Weyl's trick to (12) when $\sigma=\tau=\sharp_{\alpha}$.

If $\sim$ is deleted and $C \geq 0$ in Corollary 5.4, then [8, Corollary 3.1] is recovered.

Further, log-majorization implies weak majorization and, in particular, trace inequalities. Thus, under the hypothesis of Corollary 5.4, we find

$$
\operatorname{Tr}\left(\left(A \sharp_{1-\alpha} B\right) C^{*}\left(A \sharp_{\alpha} B\right)^{\sim} C\right) \leq \operatorname{Tr}\left(A C^{*} B^{\sim} C\right) .
$$

This trace inequality with $\sim$ deleted and $C=I$ was observed in [4]. 
The following question naturally arises: for $A, B \geq 0$ and $C \in H_{n}^{\sim}$, does the log-majorization

$$
\left(A \sigma^{\perp} B\right) C^{*}(A \sigma B)^{\sim} C \prec_{\log } A C^{*} B^{\sim} C
$$

remains true for other operator connections $\sigma$, apart from the weighted geometric means?

\section{On Ando And Visick's inequalities for the HadAmard PRODUCT}

A weighted interpolation of Ando and Visick's result (2) inspired by [5] can now be derived. The last two corollaries of the previous section provide the link.

Let $A, B \geq 0, C \in H_{n}^{\sim}, \sigma$ be a nonzero operator connection and $\alpha \in[0,1]$. Ignoring the matrix in the middle of the double log-majorization, Corollary 5.2 can be reformulated as

$$
\prod_{i=k}^{n} \lambda_{i}\left((A \sharp B) C^{*}(A \sharp B)^{\sim} C\right) \geq \prod_{i=k}^{n} \lambda_{i}\left(\left(A \sigma^{\perp} B\right) C^{*}(A \sigma B)^{\sim} C\right), \quad k=1, \ldots, n,
$$

with equality for $k=1$. Corollary 5.4 can be written as

$$
\prod_{i=k}^{n} \lambda_{i}\left(\left(A \sharp_{1-\alpha} B\right) C^{*}\left(A \sharp_{\alpha} B\right)^{\sim} C\right) \geq \prod_{i=k}^{n} \lambda_{i}\left(A C^{*} B^{\sim} C\right), \quad k=1, \ldots, n,
$$

with equality for $k=1$. On the other hand, if $D \in M_{n}$ is a diagonal matrix, we have

$$
D(A \circ B) \bar{D} \geq D((A \sharp B) \circ(A \sharp B)) \bar{D}=(D(A \sharp B) \bar{D}) \circ(A \sharp B)
$$

and corresponding eigenvalue inequalities involving such matrices hold. Consider Ando and Visick's result (2) and its parallel one without transpose [1, 12], written in the condensed form

$$
\prod_{i=k}^{n} \lambda_{i}(A \circ B) \geq \prod_{i=k}^{n} \lambda_{i}\left(A B^{\sim}\right), \quad k=1, \ldots, n
$$

where ${ }^{\sim}$ is ${ }^{T}$ or has no effect; then replace $A, B$ by $D(A \sharp B) \bar{D}, A \sharp B$, respectively, and find

$$
\prod_{i=k}^{n} \lambda_{i}((D(A \sharp B) \bar{D}) \text { о }(A \sharp B)) \geq \prod_{i=k}^{n} \lambda_{i}\left((A \sharp B) \bar{D}(A \sharp B)^{\sim} D\right), \quad k=1, \ldots, n .
$$

The previous inequalities can be complemented, using (18), (19) with $\sigma=\sharp_{\alpha}$ and replacing $C$ by a diagonal matrix $D$, which is assumed real when $\sim$ is omitted, and (20) as follows:

$$
\begin{aligned}
\prod_{i=k}^{n} \lambda_{i}\left((A \circ B)|D|^{2}\right) \geq \prod_{i=k}^{n} \lambda_{i}\left((A \sharp B) \bar{D}(A \sharp B)^{\sim} D\right) & \geq \prod_{i=k}^{n} \lambda_{i}\left(\left(A \sharp_{1-\alpha} B\right) \bar{D}(A \sharp \alpha B)^{\sim} D\right) \\
& \geq \prod_{i=k}^{n} \lambda_{i}\left(A \bar{D} B^{\sim} D\right)
\end{aligned}
$$

for $k=1, \ldots, n$. If $\sim$ is deleted and $D=I$, we get a previous result by Hiai and Lin [5] and the first inequality in (21) also gives [1, Theorem 2]. If $\sim$ is the transpose and $D=I$, we find the next parallel weighted refinement of (2).

Proposition 6.1. If $A, B \geq 0$ and $\alpha \in[0,1]$, then

$$
\prod_{i=k}^{n} \lambda_{i}(A \circ B) \geq \prod_{i=k}^{n} \lambda_{i}\left((A \sharp B)(A \sharp B)^{T}\right) \geq \prod_{i=k}^{n} \lambda_{i}\left(\left(A \sharp_{1-\alpha} B\right)\left(A \sharp_{\alpha} B\right)^{T}\right) \geq \prod_{i=k}^{n} \lambda_{i}\left(A B^{T}\right)
$$

for $k=1, \ldots, n$, occurring equality for $k=1$ in the last two inequalities. 
The first inequality in (21) holds for any $D \in M_{n}$ diagonal, but

$$
\prod_{i=k}^{n} \lambda_{i}\left((A \circ B)|D|^{2}\right) \geq \prod_{i=k}^{n} \lambda_{i}\left((A \sharp B) D^{*}(A \sharp B)^{\sim} D\right), \quad k=1, \ldots, n,
$$

does not remain true, in general, when $D$ is replaced by any $C \in H_{n}^{\sim}$.

Example 6.2. Consider

$$
A=\left[\begin{array}{ll}
1 & 0 \\
0 & 1
\end{array}\right], \quad B=\left[\begin{array}{ll}
2 & 1 \\
1 & 1
\end{array}\right] \geq 0, \quad C=\left[\begin{array}{cc}
1 & 1+i \\
1-i & -3
\end{array}\right] \in H_{2} .
$$

In this case, $A \sharp B=B^{\frac{1}{2}}$ and (22) with $\sim$ deleted and $C$ in the place of $D$ does not hold, because

$$
\lambda_{2}\left((A \circ B) C^{2}\right) \approx 3,783 \leq \lambda_{2}\left(B^{\frac{1}{2}} C\right)^{2} \approx 4,095 .
$$

\section{Another extension of Singular value inequalities By Zou}

Theorem 7.1. Let $A, B \geq 0, r \in \mathbb{N}_{0}$ and $\sigma$ be a nonzero operator connection. If $\sigma \leq \sharp$, then

$$
\lambda_{1}(A \sigma B)^{2(r+1)} \leq \lambda_{1}\left(A(A \sigma B)^{r} B(A \sigma B)^{r}\right) \leq \lambda_{1}(A B)^{r+1} .
$$

Proof. The first inequality in (23) follows from (13) with $C=(A \sigma B)^{r}$. Concerning the second one, suppose $A>0$ and $\lambda_{1}(A B)^{r+1} \leq 1$. Then $B \leq A^{-1}$, which implies

$$
(A \sigma B) B(A \sigma B) \leq(A \sigma B) A^{-1}(A \sigma B)=A^{\frac{1}{2}}\left(f_{\sigma}\left(A^{-\frac{1}{2}} B A^{-\frac{1}{2}}\right)\right)^{2} A^{\frac{1}{2}} \leq B,
$$

because $f_{\sigma}^{2}(t) \leq t, t>0$. By induction on $r \in \mathbb{N}_{0}$, we prove that $(A \sigma B)^{r} B(A \sigma B)^{r} \leq B$. This holds trivially for $r=0$. Suppose this is true for a given exponent $r$. Then

$$
(A \sigma B)^{r+1} B(A \sigma B)^{r+1}=(A \sigma B)\left((A \sigma B)^{r} B(A \sigma B)^{r}\right)(A \sigma B) \leq(A \sigma B) B(A \sigma B) \leq B,
$$

where the first inequality is a consequence of the inductive hypothesis and the second one follows from (24). Therefore $(A \sigma B)^{r} B(A \sigma B)^{r} \leq A^{-1}$ and we conclude that

$$
\lambda_{1}\left(A(A \sigma B)^{r} B(A \sigma B)^{r}\right) \leq 1 .
$$

Thus, we find the last inequality in (23). If $A \geq 0$ is not invertible, then use a continuity argument, replacing $A$ by $A+\epsilon I, \epsilon>0$.

Corollary 7.2. If $A, B \geq 0$ and $r \in \mathbb{N}_{0}$, then

$$
(A \sharp B)^{r+1} \prec_{\log }\left|A^{\frac{1}{2}}(A \sharp B)^{r} B^{\frac{1}{2}}\right| \prec_{\log }(A B)^{\frac{r+1}{2}} .
$$

Proof. Let $\sigma=\sharp$ in Theorem 7.1 and apply Weyl's trick. The obtained log-majorization implies the log-majorization between the square roots of the matrices of the previous one.

For $A, B \geq 0$ and $r \in \mathbb{N}_{0}$, the last log-majorization and (15) yield

$$
\prod_{i=1}^{k} s_{i}\left(A^{\frac{1}{2}}(A \sharp B)^{r} B^{\frac{1}{2}}\right) \leq \prod_{i=1}^{k} s_{i}^{\frac{r+1}{2}}(A B), \quad k=1, \ldots, n .
$$

For $r=1$, these are the singular value inequalities proved by Zou in [14, Theorem 2.10].

\section{Acknowledgements}

The authors are grateful to the referee for careful reading of the manuscript. The work of the first author is supported by the Center for Research and Development in Mathematics and Applications (CIDMA) through the Portuguese Foundation for Science and Technology (FCT - Fundação para a Ciência e a Tecnologia), within the projects UIDB/04106/2020 
and UIDP/04106/2020. The research of the second author was partially financed by Portuguese funds through FCT (Fundação para a Ciência e a Tecnologia), within the projects UIDB/00013/2020 and UIDP/00013/2020.

\section{REFERENCES}

[1] T. Ando, Majorization relations for Hadamard products, Linear Algebra Appl. 223/224 (1995) 57-64.

[2] R. Bhatia, Matrix Analysis, Springer, New York, 1997.

[3] R. Bhatia, Positive definite matrices, Princeton University Press, USA, 2007.

[4] R. Bhatia, Y. Lim and T. Yamazaki, Some norm inequalities for matrix means, Linear Algebra Appl. 501 (2016), 112-122.

[5] F. Hiai and M. Lin, On an eigenvalue inequality involving the Hadamard product, Linear Algebra Appl. 515 (2017), 313-320.

[6] R. A. Horn and C. R. Johnson, Matrix Analysis, Cambridge University Press, Cambridge, 1985.

[7] F. Kubo and T. Ando, Means of positive linear operators, Math. Ann. 246 (1980), 205-224.

[8] R. Lemos and G. Soares, Some log-majorizations and an extension of a determinantal inequality, Linear Algebra Appl. 547 (2018), 19-31.

[9] K. Löwner, Über monotone Matrixfunktionen, Math. Z. 38 (1934), 177-216.

[10] A. W. Marshall and I. Olkin, Inequalities: Theory of Majorization and its Applications, Academic, New York, 1979.

[11] Y. Udagawa, S. Wada, T. Yamazaki and M. Yanagida, On a family of operator means involving the power difference means, Linear Algebra Appl. 485 (2015), 124-131.

[12] G. Visick, A weak majorization involving the matrices $A$ o $B$ and $A B$, Linear Algebra Appl. 223/224 (1995), $731-744$.

[13] X. Zhan, Matrix inequalities, Lecture Notes in Mathematics 1790, Springer, Berlin, 2002.

[14] L. Zou, An arithmetic geometric mean inequality for singular values and its applications, Linear Algebra Appl. 528 (2017), 25-32.

(R. Lemos) Cidma, Mathematics Department, Aveiro University, 3810-193 Aveiro, Portugal E-mail address: rute@ua.pt

(G. Soares) Mathematics Center CMAT, Pole CMAT-UtAd, Universidade de Trás-os-Montes e Alto Douro, Escola das Ciências e Tecnologia, Quinta dos Prados, 5000-801 Vila Real, Portugal

E-mail address: gsoares@utad.pt 